\title{
Factors affecting discontinuation of initial treatment with paroxetine in panic disorder and major depressive disorder
}

This article was published in the following Dove Press journal:

Neuropsychiatric Disease and Treatment

18 September 2014

Number of times this article has been viewed

\author{
Akiko Aoki' \\ Shin Ishiguro' \\ Takashi Watanabe' \\ Mikito Ueda' \\ Yuki Hayashi' \\ Kazufumi Akiyama ${ }^{2}$ \\ Kazuko Kato ${ }^{3}$ \\ Yoshimasa Inoue' \\ Shoko Tsuchimine ${ }^{4}$ \\ Norio Yasui-Furukori ${ }^{4}$ \\ Kazutaka Shimoda' \\ 'Department of Psychiatry, \\ ${ }^{2}$ Department of Biological Psychiatry \\ and Neuroscience, Dokkyo Medical \\ University School of Medicine, \\ Tochigi, Japan; ${ }^{3}$ Sakura La Mental \\ Clinic, Tochigi, Japan; ${ }^{4}$ Department \\ of Neuropsychiatry, Hirosaki \\ University Graduate School \\ of Medicine, Aomori, Japan
}

Objective: The aims of the present study were to analyze the association between discontinuation of paroxetine $(\mathrm{PAX})$ and the genetic variants of the polymorphism in the serotonin transporter gene-linked polymorphic region (5-HTTLPR) in Japanese patients with panic disorder (PD) and major depressive disorder (MDD).

Methods: The 5-HTTLPR genotype was determined by polymerase chain reaction method. PAX plasma concentration was measured by high-performance liquid chromatography to confirm adherence.

Results: When comparing between the PD and MDD patients with the chi-square test and Fisher's exact test, the PD patients had a significant and higher discontinuation rate due to nonadherence than did the MDD patients (13.5\% [7/52] versus $0 \%$ [0/88], respectively; $P<0.001$ ). MDD patients had a significant and higher discontinuation rate due to untraceability than PD patients $(12.5 \%$ [11/88] versus $1.9 \%$ [1/52]; $P=0.032)$. Multilogistic regression revealed a tendency for the long/short and short/short genotypes to affect discontinuation due to adverse effects in PD patients (25.0\% versus $6.3 \%$, respectively; $P=0.054)$.

Conclusion: The results indicate that the 5-HTTLPR genotype might contribute to the discontinuation of initial PAX treatment due to adverse effects in PD patients.

Keywords: paroxetine, discontinuation, panic disorder, major depressive disorder

\section{Introduction}

Continuation of psychopharmacotherapy is essential for the clinical outcome of depressive and anxiety disorders; however, factors that affect their discontinuation are not fully known. In clinical trials, discontinuation of antidepressants has been attributed to lack of clinical response, adverse effects, pharmacophobia in panic disorder (PD), ${ }^{1}$ and depression. ${ }^{2}$

Genetic polymorphism, which is related to adverse effects induced by the selective serotonin reuptake inhibitors (SSRIs), has not been linked to antidepressant discontinuation thus far. However, Perlis et $\mathrm{al}^{3}$ reported that the short (S) allele of serotonin transporter gene-linked polymorphic region (5-HTTLPR) might contribute to agitation and insomnia with the administration of fluoxetine. Popp et al investigated 65 subjects who were administered antidepressants with a predominant serotonergic mechanism and reported that one-half of the subjects with the $\mathrm{S} / \mathrm{S}$ genotype suffered significant adverse effects, $40 \%$ of subjects with $\mathrm{S} /$ long (L) genotype had side effects, but none of the subjects with the L/L genotype reported side effects. ${ }^{4}$ Murphy et al reported that geriatric patients with S/S genotype of 5-HTTLPR showed more intense side effects with paroxetine (PAX) than subjects with the L/L genotype, and
Correspondence: Kazutaka Shimoda Department of Psychiatry, Dokkyo Medical University School of Medicine, 880 Kitakobayashi, Mibu, Shimotsuga, Tochigi, 32I-0293, Japan

Tel $+8 \mid 28286$ IIIII

Fax +8I 282865187

Email shimoda@dokkyomed.ac.jp 
subjects with the $\mathrm{S} / \mathrm{S}$ genotype received a lower final dose of PAX and they had lower adherence than subjects with L/L genotype. ${ }^{5}$

The 5-HTTLPR has been known as a functional polymorphism. In vitro, the activity of the $\mathrm{L}$ variant is more than doubled when compared to that of the $\mathrm{S}$ variant in the 5-HTT mRNA synthesis and 5-HTT expression, ${ }^{6}$ which indicates that the 5-HTT gene transcription is affected by the 5-HTTLPR genetic polymorphism. ${ }^{6}$

Stahl previously reported that PD patients are more sensitive to SSRIs than depressed patients, given that the former can readily manifest short-term deterioration of their symptoms during the initial phase of pharmacotherapy. ${ }^{7}$ Consequently, PD patients should be initiated from a lower dose than depressed patients. ${ }^{7}$ In addition, Louie et al asserted that PD patients comorbid with major depressive disorder (MDD) showed lower tolerance to SSRIs compared to those with MDD alone. ${ }^{8}$

Against this background, the object of the present study was to investigate the association between the discontinuation of the initial PAX treatment and the genetic variants of 5-HTTLPR in Japanese patients with PD or MDD, and then to investigate the difference between these two diseases in relation to the discontinuation.

\section{Patients and methods PD patients}

Fifty-two Japanese patients who were diagnosed as having PD, according to the Diagnostic and Statistical Manual of Mental Disorders, Fourth Edition, Text Revision (DSMIV-TR) criteria, and who were administered PAX $10 \mathrm{mg} /$ day (paroxetine $\mathrm{HCl}$; Paxil ${ }^{\circledR}$; GlaxoSmithKline KK, Tokyo, Japan), were subjected to analysis in this study. All PD patients (20 men, 32 women; age, 33.9 \pm 8.3 years [mean \pm standard deviation]; range of age, 20-58 years) were outpatients at Dokkyo Medical University Hospital (located in Mibu, Tochigi, Japan) and the Sakura La Mental Clinic (located in Utsunomiya, Tochigi, Japan). Body weight ranged from $40-95 \mathrm{~kg}(57.9 \pm 11.4 \mathrm{~kg})$. The PD patients in this study were part of the subject group $(n=65)$ in our previous study. ${ }^{9}$ Out of 65 patients, 52 subjects with PD without MDD were selected, and their raw data from the clinical evaluations and the genotyping of 5-HTTLPR were used and reanalyzed in the present study.

Patients were permitted to take low doses of lorazepam $(<2.0 \mathrm{mg} /$ day $)$ when they had panic attacks, and patients who reported insomnia were allowed to be prescribed brotizolam 0.25 or $0.5 \mathrm{mg}$ at bedtime. The severity of PD was assessed by using the Panic and Agoraphobia Scale observer-rated version ${ }^{10}$ before initiating pharmacotherapy with PAX.

Patients were excluded from the present study if they had: 1) diagnosis of axis I other than PD; 2) diagnosis of axis II; 3) severe general medical condition or major abnormal laboratory test; 4) suicide risk; 5) history of substance abuse; 6) use of the 5-hydroxytryptamine (5-HT) agonist, benzodiazepines, antidepressants, antipsychotics before study entry; or 7) pregnancy. Written informed consent was obtained from all PD subjects. The present study was approved by the ethics committees of Dokkyo Medical University Hospital and the Sakura La Mental Clinic.

\section{MDD patients}

In addition, 88 patients who were diagnosed as having MDD, according to the DSM-IV-TR criteria and who were treated with PAX $20 \mathrm{mg} /$ day for 2 weeks as initial treatment. All MDD subjects (29 men, 59 women; age, 46.8 \pm 13.6 years; range of age, 20-69 years) were outpatients at Hirosaki University Hospital (located in Hirosaki, Aomri, Japan). The 88 MDD patients were part of the subject group $(n=120)$ in an earlier study. ${ }^{11}$ Out of 120 subjects, 88 subjects with MDD whose genotype of 5-HTTLPR were selected, and their raw data were used and reanalyzed in the present study. Body weight ranged from $40-90 \mathrm{~kg}(56.3 \pm 10.0 \mathrm{~kg})$. Clinical assessment by using the Montgomery-Åsberg Depression Rating Scale $^{12}$ was conducted before initiating pharmacotherapy. No other psychotropic drugs were administered other than diazepam (2-5 mg/day) for anxiety and brotizolam $(0.25$ $\mathrm{mg} /$ day) for insomnia.

Patients were excluded if they had: 1) diagnosis of axis I other than MDD; 2) diagnosis of axis II; 3) severe general medical condition or major abnormal laboratory test; 4) suicide risk; 5) history of substance abuse; 6) use of 5-HT agonist, benzodiazepines, antidepressants, antipsychotics, before study entry; or 7) pregnancy. The present study was approved by the Ethics Committee of Hirosaki University Hospital. Written informed consent was obtained from the patients.

\section{Assessment of discontinuation of pharmacotherapy}

Patients who stopped taking PAX due to adverse effects were categorized as "discontinuation due to adverse effects." Patients whose plasma PAX concentration was below the limit of detection were categorized as "discontinuation due to nonadherence." Patients who did not 
visit the hospital 2 weeks after starting PAX (ie, lost to follow-up) were categorized as "discontinuation due to untraceability."

\section{Determination of PAX plasma concentrations}

PAX plasma concentration was determined by high-performance liquid chromatography. ${ }^{13}$ The lowest limit of detection was 0.5 $\mathrm{ng} / \mathrm{mL}$, and the interassay coefficient of variation was $<5 \%$ at a PAX concentration of $1 \mathrm{ng} / \mathrm{mL}$.

\section{Genotyping}

The L and S alleles of 5-HTTLPR were determined by polymerase chain reaction as reported by Lesch et al ${ }^{14}$ and Heils et al. ${ }^{6}$

\section{Statistical analysis}

To examine the relationship between the demographic variables and the discontinuation of PAX treatment, multilogistic regression analysis was conducted. For comparison of the discontinuation rates between MDD patients and PD patients, the chi-square test or the Fisher's exact test (when data were sparse) was carried out. For comparison of the differences of demographic data between MDD patients and PD patients, the Student's $t$-test, Welch's $t$-test, and chi-square test, were used. Multilogistic regression analysis, chi-square test, Fisher's exact test, Welch's $t$-test, and Student's $t$-test were conducted with Dr SPSS II for Windows (SPSS Japan Inc., Tokyo, Japan) and IBM SPSS statistics version 19.0 (Japan IBM, Tokyo, Japan). $P$-values of $<0.05$ were considered significant.

\section{Results}

\section{Comparison between PD and MDD}

To investigate the relationship between the discontinuation of PAX treatment in all patients (ie, PD plus MDD) and demographic variables, multilogistic regression analysis was performed with total discontinuation due to all reasons, discontinuation due to adverse effects, discontinuation due to nonadherence, and discontinuation due to untraceability as the dependent variables and age, sex, body weight, diagnosis of PD or MDD, and L/S or S/S genotype of 5-HTTLPR as independent variables. No significant relationships were found between any of the independent variables and discontinuation due to adverse effects, discontinuation due to nonadherence, discontinuation due to untraceability, or total discontinuation (ie, nonadherence plus adverse effect plus untraceability).
In the comparison between PD and MDD using the chi-square test and Fisher's exact test (Table 1), PD patients had a significant and higher discontinuation rate due to nonadherence than MDD patients (13.5\% [7/52] versus $0 \%$ [0/88], respectively; $P<0.001)$. MDD patients had a significant and higher discontinuation rate due to untraceability than $\mathrm{PD}$ patients $(12.5 \%$ [11/88] versus $1.9 \%$ [1/52]; $P=0.032)$. There was no significant difference between $\mathrm{PD}$ and MDD patients in the discontinuation rate due to adverse effects $(13.5 \%$ [7/52] versus $6.8 \%[6 / 88] ; P=0.233)$ or discontinuation due to all reasons $(28.8 \%$ [15/52] versus $19.3 \%$ [17/88]; $P=0.216)$.

\section{Characteristics of PD patients}

PD patient demographics are described in Table 2. The mean body weight of patients with the L/S genotype was significant and higher compared to subjects with the $\mathrm{S} / \mathrm{S}$ genotype of 5-HTTLPR $(P=0.026)$. No significant differences were observed in sex, age, number of panic attacks/week, or the Panic and Agoraphobia Scale score at baseline between patients with the $\mathrm{L} / \mathrm{S}$ genotype and those with the $\mathrm{S} / \mathrm{S}$ genotype.

Of the 52 PD patients enrolled, seven patients (13.5\%) stopped taking PAX due to adverse effects, such as dry mouth, nausea, and diarrhea (discontinuation due to adverse effects). These seven patients reported that they stopped taking PAX because of adverse effects, but they consented to provide blood samples for determination of genotype. One patient did not come to the outpatient clinic (discontinuation due to untraceability). Additionally, seven patients (13.5\%) had PAX plasma concentrations below the detection limit (discontinuation due to nonadherence). Thus, 15 patients discontinued, and 37 patients continued with PAX (Table 3).

Table I Comparison of discontinuation of PAX treatment between patients with PD and MDD

Discontinued, $\mathbf{n}(\%)$

\begin{tabular}{ll}
\hline Total discontinuation rate & \\
PD & $15 / 52(28.8)$ \\
MDD & $17 / 88(19.3)$ \\
Discontinuation rate due to nonadherence & \\
PD & $7 / 52(13.5)^{* * \pi} \pi$ \\
MDD & $0 / 88(0)^{* * \pi}$ \\
Discontinuation rate due to adverse effects & \\
PD & $7 / 52(13.5)$ \\
MDD & $6 / 88(6.8)$ \\
Discontinuation rate due to untraceability & \\
PD & $1 / 52(1.9)^{* \pi, \pi}$ \\
MDD & $11 / 88(12.5)^{* \pi}$
\end{tabular}

Notes: TFisher's exact test. $* P<0.05$. $* * P<0.001$.

Abbreviations: PD, panic disorder; MDD, major depressive disorder; PAX, paroxetine. 
Table 2 Demographic characteristics of PD patients at baseline, according to 5-HTTLPR genotype

\begin{tabular}{llll}
\hline & 5-HTT gene-linked polymorphic region & Total \\
\hline Genotype & L/S genotype & S/S genotype & 52 \\
\# of patients & 20 & 32 & $20 / 32$ \\
Male/female, $\mathrm{n}$ & $9 / I \mathrm{II} / 2 \mathrm{I}$ & $33.9 \pm 8.3$ \\
Age, years & $36.0 \pm 6.5$ & $32.7 \pm 9.2$ & $57.9 \pm I I .4$ \\
Body weight, $\mathrm{kg}$ & $62.3 \pm 12.8$ & $55.1 \pm 9.7$ & $39 / \mathrm{I3}$ \\
With/without agoraphobia, $\mathrm{n}$ & $13 / 7$ & $26 / 6$ & $2.8 \pm 3.2$ \\
Panic attacks per week, $\mathrm{n}$ & $2.7 \pm 2.5$ & $2.9 \pm 3.7$ & $21.5 \pm 6.5$ \\
Panic and Agoraphobia Scale score & $20.5 \pm 7.5$ & $22.2 \pm 5.8$ & \\
\hline
\end{tabular}

Notes: Values are numbers of subjects or mean $\pm S D$.

Abbreviations: PD, panic disorder; L/S, long/short; S/S, short/short; SD, standard deviation.

Multilogistic regression analysis was conducted to examine the relationship between demographic variables and discontinuation of PAX treatment, with discontinuation due to nonadherence, discontinuation due to adverse effects, discontinuation due to untraceability, and total discontinuation as the dependent variables and age, sex, body weight, comorbid agoraphobia, and L/S or S/S genotype of 5-HTTLPR as the independent variables. No significant relationships were found between any of the independent variables and total discontinuation due to all reasons, discontinuation due to nonadherence, or discontinuation due to untraceability. The multilogistic regression analysis revealed a tendency for the L/S genotype to be associated with higher discontinuation rate due to adverse effects in PD patients compared to those with S/S genotype (25.0\% versus $6.3 \%$, respectively; $P=0.054$ ).

\section{Characteristics of MDD patients}

MDD patient demographics are shown in Table 4. There were no subjects with the L/L genotype. No significant differences

Table 3 Discontinuation of pharmacotherapy with PAX at 2 weeks after initiation, according to 5-HTTLPR genotype in PD patients

\begin{tabular}{ll}
\hline & Discontinued \\
\hline Total discontinuation rate & \\
L/S genotype & $8(8 / 20=40 \%)$ \\
S/S genotype & $7(7 / 32=21.9 \%)$ \\
Discontinuation rate due to nonadherence & \\
L/S genotype & $3(3 / 20=15 \%)^{\pi}$ \\
S/S genotype & $4(4 / 32=12.5 \%)^{\pi}$ \\
Discontinuation rate due to adverse effects & \\
L/S genotype & $5(5 / 20=25.0 \%)^{* \pi}$ \\
S/S genotype & $2(2 / 32=6.3 \%)^{* \pi}$ \\
Discontinuation rate due to untraceability & \\
L/S genotype & $0(0 / 20=0 \%)^{\pi}$ \\
S/S genotype & $1(I / 32=3.1 \%)^{\pi}$ \\
\hline
\end{tabular}

Notes: 'TFisher's exact test. $* P=0.067$.

Abbreviations: PAX, paroxetine; PD, panic disorder; L/S, long/short; S/S, short/ short. were seen in age, sex, body weight, or initial MontgomeryÅsberg Depression Rating Scale score between the patients with the L/S genotype and patients with $\mathrm{S} / \mathrm{S}$ genotype before starting PAX pharmacotherapy.

None of the patients discontinued due to nonadherence (Table 5), six discontinued due to adverse effects, including nausea and delirium. Also, eleven did not come to the outpatient clinic (discontinuation due to untraceability).

Accordingly, 17 patients discontinued, and 71 patients continued with PAX. Multilogistic regression analysis was conducted to investigate the relationship between discontinuation of PAX, discontinuation due to adverse effects, discontinuation due to untraceability, and total discontinuation as the dependent variables and age, sex, body weight, and L/S or S/S genotype of 5-HTTLPR as the independent variables. No significant relationships were found between any of the dependent and independent variables.

\section{Discussion}

Discontinuation rate of SSRIs has been reported to be $16.8 \%-31.0 \%$ in MDD. ${ }^{15}$ Goethe et al reported that the discontinuation rate was $26.9 \%$, and discontinuation rate did not differ among SSRIs. ${ }^{16}$ Total discontinuation rate in PD and MDD in the present study was $22.8 \%$, which is concordant with the previous report. ${ }^{15,16}$

Table 4 Demographic characteristics of MDD patients at baseline, according to 5-HTTLPR genotype

\begin{tabular}{llll}
\hline & 5-HTTLPR & & Total \\
\hline Genotype & L/S genotype & S/S genotype & \\
\# of patients & 31 & 57 & 88 \\
Male/female, $\mathrm{n}$ & $10 / 21$ & $19 / 38$ & $29 / 59$ \\
Age, years & $46.0 \pm 12.6$ & $47.3 \pm 14.2$ & $46.8 \pm 13.6$ \\
Body weight, $\mathrm{kg}$ & $56.7 \pm 9.1$ & $56.0 \pm 10.5$ & $56.3 \pm 10.0$ \\
MADRS score & $40.5 \pm 9.3$ & $38.0 \pm 9.5$ & $38.9 \pm 9.5$ \\
\hline
\end{tabular}

Note: Values are numbers or mean $\pm S D$.

Abbreviations: MDD, major depressive disorder; MADRS: Montgomery-Åsberg Depression Rating Scale; SD, standard deviation; L/S, long/short; S/S, short/short. 
Table 5 Discontinuation of pharmacotherapy with PAX at 2 weeks after initiation, according to 5-HTTLPR genotype in MDD patients

\begin{tabular}{ll}
\hline & Discontinued \\
\hline $\begin{array}{l}\text { Total discontinuation rate } \\
\text { L/S genotype }\end{array}$ & $4(4 / 3|=| 2.9 \%)^{\pi}$ \\
S/S genotype & $13(\mid 3 / 57=22.8 \%)^{\pi}$ \\
Discontinuation rate due to nonadherence & \\
L/S genotype & $0(0 / 3 \mid=0 \%)^{\pi}$ \\
S/S genotype & $0(0 / 57=0 \%)^{\pi}$ \\
Discontinuation rate due to adverse effects & \\
L/S genotype & $I(I / 3 \mid=3.2 \%)^{\pi}$ \\
S/S genotype & $5(5 / 57=8.8 \%)^{\pi}$ \\
Discontinuation rate due to untraceability & \\
L/S genotype & $3(3 / 3 \mid=9.7 \%)^{\pi}$ \\
S/S genotype & $8(8 / 57=\mid 4.0 \%)^{\pi}$ \\
\hline
\end{tabular}

Note: "Fisher's exact test.

Abbreviations: PAX, paroxetine; MDD, major depressive disorder; L/S, long/short; S/S, short/short.

Stahl previously reported that patients with PD are typically initiated from a lower dose of SSRIs compared to depressed patients because their symptoms can easily worsen over the short term, worsening at the start of pharmacotherapy. ${ }^{7}$ Toni et al reported a $10.6 \%$ discontinuation rate for antidepressants (PAX, imipramine, and clomipramine) due to adverse effects in a 3-year longitudinal study of $\mathrm{PD},{ }^{1}$ which is very similar to that in $\mathrm{PD}(13.5 \%)$ in the present study. When taking the difference in daily dose of PAX between PD $(10 \mathrm{mg} /$ day $)$ and MDD (20 mg/day) patients into account, PD patients might be more intolerant of PAX in the initial 2 weeks of treatment than MDD patients; indeed, the present results showed that they can experience adverse effects easily in this initial phase of antidepressant treatment.

Freire et al evaluated and analyzed personality traits in subjects with: PD without MDD; those with MDD without PD; those with PD and MDD; and control group by Maudsley Personality Inventory. ${ }^{17}$ They reported that those with MDD without PD, and those with PD and MDD had significant lower extraversion score compared to control subjects, while a significant difference in extraversion score was not observed between subjects with PD without MDD and control subjects. Such difference in extraversion score might explain the behavioral difference under pharmacotherapy with PAX between two disorders in the present study. That is, when the treatment is not beneficial because of the lack of efficacy and/or adverse effects, MDD patients tend to stop the treatment without consultation with their doctor in charge (ie, untraceability) because of their introversion tendency; however, PD patients tend to pretend to be adherent with pharmacotherapy without taking their drugs (ie, nonadherence).
A meta-analysis of 26 studies reported the trend of association between the 5-HTTLPR S allele and the increased scores of anxiety-related personality trait $(P=0.087){ }^{18}$ Yamakawa et al found that among 264 women with metabolic disease, those with S/S genotype of 5-HTTLPR showed significantly reduced fasting blood glucose following nutritional intervention both over the short (11 weeks) and long (23 years) term, ${ }^{19}$ although they stopped short of confirming an association between adherence to the intervention and 5-HTTLPR polymorphism. Thus, the S/S genotype of the 5-HTTLPR might be associated with anxiety-related personality trait, which leads patients to favorable adherence; however, such association was not observed in the present study.

Whether 5-HTTLPR might contribute to the risk of adverse effects with SSRIs is uncertain; however, Perlis et al asserted that the $\mathrm{S}$ allele of 5-HTTLPR might contribute to the risk of insomnia and agitation with fluoxetine treatment in patients with MDD, ${ }^{3}$ while no such association was seen in MDD patients in the present study. One possible explanation is the difference of duration of observation (12 weeks in the report from Perlis et $\mathrm{al}^{3}$ and 2 weeks in the present study). The results of the present study also indicate that discontinuation rate in the subjects with the L/S genotype of 5-HTTLPR showed a higher tendency of discontinuation rate due to adverse effects compared to those with S/S genotype in PD, while no such association was seen in MDD patients in the present study.

Of course, there are several limitations that should be mentioned in the present study. First, duration of observation was relatively short, ie, 2 weeks. Second, numbers of the subjects in both of PD and MDD group were relatively small. The power of each statistical analysis was calculated and yielded values ranging from $0.05-0.746$, which suggests no statistical significant differences are associated with low statistical power, which might result in type II error.

Additionally, the CYP2D6 genotype has significant impact on pharmacokinetics of PAX even in Asian population, ${ }^{20,21}$ which means activity of CYP2D6 is one of the confounding factors; however, the CYP2D6 genotype has not been determined in the subjects in the present study. Our preliminary findings should be replicated in a larger number of subjects and under longer duration of observation to draw firm conclusion.

\section{Conclusion}

The findings of the present study indicate that 5-HTTLPR genotype might be one of the factors that are related to the discontinuation of initial PAX treatment due to adverse 
effects in PD patients. The findings also indicate that PD patients may be more intolerant than MDD patients to PAX treatment within the first 2 weeks.

\section{Acknowledgments}

The present study funded by a Grant-in-Aid for Scientific Research (KAKENHI) for the Japan Society for the Promotion of Research (\#25461739) and the Seki Minato Memorial Awards to Kazutaka Shimoda. The funding sources played no role in: the planning of the study design; collection or analysis of data; interpretation of results; writing of the manuscript; or decision to submit the paper for publication.

\section{Disclosure}

Kazutaka Shimoda has received research support from: Shionogi \& Co., Ltd.; Eli Lilly Japan, KK; Yoshitomi Pharmaceutical Industries, Ltd.; Meiji Seika Pharma Co., Ltd.; Eisai Co., Ltd.; Pfizer Inc.; GlaxoSmithKline KK; Otsuka Pharmaceutical Co., Ltd.; Daiichi Sankyo Co.; and Takeda Pharmaceutical Co., Ltd. Kazutaka Shimoda also received honoraria from: Kowa Pharmaceutical Co. Ltd.; Mitsubishi Tanabe Pharma Corporation; Meiji Seika Pharma Co., Ltd.; Dainippon Sumitomo Pharma Co., Ltd.; Ono Pharmaceutical Co., Ltd.; GlaxoSmithKline KK; and Eisai Co., Ltd. The other authors report no conflicts of interest in this work.

\section{References}

1. Toni C, Perugi G, Frare F, Mata B, Akiskal HS. Spontaneous treatment discontinuation in panic disorder patients treated with antidepressants. Acta Psychiatr Scand. 2004;110(2):130-137.

2. Johnson DA. Depression: treatment compliance in general practice. Acta Psychiatr Scand Suppl. 1981;290:447-453.

3. Perlis RH, Mischoulon D, Smoller JW, et al. Serotonin transporter polymorphisms and adverse effects with fluoxetine treatment. Biol Psychiatry. 2003;54(9):879-883.

4. Popp J, Leucht S, Heres S, Steimer W. Serotonin transporter polymorphisms and side effects in antidepressant therapy - a pilot study. Pharmacogenomics. 2006;7(2):159-166.

5. Murphy GM, Hollander SB, Rodrigues HE, Kremer C, Schatzberg AF. Effects of the serotonin transporter gene promoter polymorphism on mirtazapine and paroxetine efficacy and adverse events in geriatric major depression. Arch Gen Psychiatry. 2004;61(11):1163-1169.
6. Heils A, Teufel A, Petri S, et al. Allelic variation of human serotonin transporter gene expression. J Neurochem. 1996;66(6):2621-2624.

7. Stahl SM. Essential Psychopharmacology: Neuroscientific Basis and Practical Applications. 2nd ed. New York: Cambridge University Press; 2000.

8. Louie AK, Lewis TB, Lannon RA. Use of low-dose fluoxetine in major depression and panic disorder. J Clin Psychiatry. 1993;54(11):435-438.

9. Ishiguro $\mathrm{S}$, Watanabe $\mathrm{T}$, Ueda $\mathrm{M}$, et al. Determinants of pharmacodynamic trajectory of the therapeutic response to paroxetine in Japanese patients with panic disorder. Eur J Clin Pharmacol. 2011;67(12):1213-1221.

10. Bandelow B. Assessing the efficacy of treatments for panic disorder and agoraphobia. II. The Panic and Agoraphobia Scale. Int Clin Psychopharmacol. 1995;10(2):73-81.

11. Yasui-Furukori N, Nakagami T, Kaneda A, et al. Inverse correlation between clinical response to paroxetine and plasma drug concentration in patients with major depressive disorders. Hum Psychopharmacol. 2011;26(8):602-608.

12. Montgomery SA, Asberg M. A new depression scale designed to be sensitive to change. Br J Psychiatry. 1979;134:382-389.

13. Hikida K, Inoue Y, Nouchi E, Ohkura Y. Determination of etizolam in human serum or plasma using automated column-switching highperformance liquid chromatography. Japanese Journal of Clinical Chemistry. 1990;19(4):354-359.

14. Lesch KP, Bengel D, Heils A, et al. Association of anxiety-related traits with a polymorphism in the serotonin transporter gene regulatory region. Science. 1996;274(5292):1527-1531.

15. Gartlehner G, Hansen RA, Carey TS, Lohr KN, Gaynes BN, Randolph LC. Discontinuation rates for selective serotonin reuptake inhibitors and other second-generation antidepressants in outpatients with major depressive disorder: a systematic review and meta-analysis. Int Clin Psychopharmacol. 2005;20(2):59-69.

16. Goethe JW, Woolley SB, Cardoni AA, Woznicki BA, Piez DA. Selective serotonin reuptake inhibitor discontinuation: side effects and other factors that influence medication adherence. J Clin Psychopharmacol. 2007;27(5):451-458

17. Freire RC, Lopes FL, Veras AB, et al. Personality traits spectrum in panic disorder and major depression. Rev Bras Psiquiatr. 2007;29(1):31-34.

18. Sen S, Burmeister M, Ghosh D. Meta-analysis of the association between a serotonin transporter promoter polymorphism (5-HTTLPR) and anxiety-related personality traits. Am JMed Genet B Neuropsychiatr Genet. 2004;127B(1):85-89.

19. Yamakawa M, Fukushima A, Sakuma K, Yanagisawa Y, Kagawa Y. Serotonin transporter polymorphisms affect human blood glucose control. Biochem Biophys Res Commun. 2005;334(4):1165-1171.

20. Sawamura K, Suzuki Y, Someya T. Effects of dosage and CYP2D6mutated allele on plasma concentration of paroxetine. Eur J Clin Pharmacol. 2004;60(8):553-557.

21. Ueda M, Hirokane G, Morita S, et al. The impact of CYP2D6 genotypes on the plasma concentration of paroxetine in Japanese psychiatric patients. Prog Neuropsychopharmacol Biol Psychiatry. 2006;30(3): 486-491.
Neuropsychiatric Disease and Treatment

\section{Publish your work in this journal}

Neuropsychiatric Disease and Treatment is an international, peerreviewed journal of clinical therapeutics and pharmacology focusing on concise rapid reporting of clinical or pre-clinical studies on a range of neuropsychiatric and neurological disorders. This journal is indexed on PubMed Central, the 'PsycINFO' database and CAS,
Dovepress

and is the official journal of The International Neuropsychiatric Association (INA). The manuscript management system is completely online and includes a very quick and fair peer-review system, which is all easy to use. Visit http://www.dovepress.com/testimonials.php to read real quotes from published authors. 